\title{
Epistemología y cibernética
}

\author{
Ignasi Brunet Icart
}

Universitat Rovira i Virgili. Departament de Gestió d'Empreses

Av. Universitat, 1. 42204, Reus (Tarragona) Spain

ibic@fcee.urv.es

\section{Antoni Morell Blanch}

Universitat de Lleida. Departament d'Economia Aplicada

Pl. Víctor Siurana, 1. 25003 Lleida

antonio.morell@econap.udl.es

Data de recepció: febrer 2001

Data d'acceptació: febrer 2001

\section{Resumen}

Si el observador y el observado forman parte del mismo objeto descrito, la convergencia entre el sujeto cognoscente y el objeto por conocer no puede sustraerse del principio de reflexividad, que propicia una mejor y más profunda solidez del corpus científico generado. La superioridad del principio de reflexividad se fundamenta en la naturaleza autorreferente de los sistemas sociales, esto es, en la capacidad que desarrollan los sistemas para tomar conciencia de sí y delimitarse respecto a un entorno o ambiente. A través de tal delimitación los sistemas acceden a la autoobservación, sin ella los sistemas no son capaces de generar todos los componentes que precisan para sus operaciones, esto es, no son capaces de llevar a cabo sus procesos autopoiéticos.

Palabras clave: autorreflexividad. cibernética, epistemología, sujeto/objeto, biología.

\section{Resum}

Si observador i observat formen part del mateix objecte descrit, la convergència entre el subjecte i l'objecte no pot ignorar el principi de reflexivitat, que proporciona una solidesa millor del corpus científic generat. La superioritat del principi de reflexivitat es fonamenta en la naturalesa autoreferent dels sistemes socials, és a dir, en la capacitat que desenvolupen els sistemes per tal de prendre consciència d'ells mateixos i delimitar-se respecte a un entorn o ambient. Mitjançant tal delimitació els sistemes accedeixen a l'autoobservació, sense aquesta els sistemes són incapaços de generar tots els components que requereixen per a les seves operacions, és a dir, no són capaços de dur a terme els seus processos autopoiètics.

Paraules clau: autoreflexivitat, cibernètica, epistemologia, subjecte/objecte, biologia.

\section{Abstract. Epistemology and cybernetics}

If the observer and the observatet belong to the same described object, the convergence between the subject can't ignore the principle of refleivity that gives a better understanding if the generated scientific corpus. The superiority of the reflexivity principle is based in the self-referential nature of the social systems; in the capacity that the systems develop in order to lose conscience of themselves, and delimit themselves from the surrounding and 
environment. Through that delimitation the systems gain access to self-observation; without this the system are incapable to generate all the componentes required for their operations, are incapable of performing their autopoietic processes.

Key words: self-reflexivity, cybernetic, epistemology, subject/object, biology.

\section{Sumario}

\section{Introducción 4. El conocimiento del conocimiento}

2. La cibernética de segundo orden

5. El constructivismo

3. La epistemología de G. Bateson

6. Conclusión

Bibliografía

\section{Introducción}

La epistemología como reflexión acerca de lo que las disciplinas científicas están produciendo, trata de evaluar la naturaleza y calidad de su conocimiento científico, la verdad o falsedad de sus teorías o cómo proveen de explicaciones adecuadas o cuál es la estructura formal y conceptual de sus teorías o qué relación debe darse entre la explicación y la predicción de un fenómeno, además de plantear el problema de la elección entre diversos métodos y, como no, interrogarse acerca de la naturaleza de las regularidades y leyes científicas. De ahí que la reflexión epistemológica asuma dos tareas: 1) examinar críticamente la práctica científica y llamar la atención sobre las dificultades que aparecen en esta práctica (función crítica), y 2) desarrollar una concepción sistemática alternativa a la práctica científica aplicada que implique alguna mejora en la resolución de los problemas de los que se ocupa la ciencia (función heurística). Estas dos funciones plantean una reflexión sobre los límites del pensamiento y las condiciones de su ejercicio, al ser relevantes las convenciones sociales para la producción del conocimiento, dado que las categorías, los hechos y los valores no pueden ser separados de manera realista.

Toda mirada sobre la realidad es un acto de selección, de construcción y de interpretación que se hace desde un sujeto en un contexto, Mirada que es anterior, y posterior, al trabajo de organización técnica de las unidades operacionales. No es de extrañar, pues, que, en contra de la reflexión epistemológica clásica, cimentada en la distancia entre el sujeto y el objeto, se constituya todo un vasto trabajo de replanteamiento y revisión que culmina en la epistemología (cibernética) de la reflexividad, cuya tesis se sintetiza en que un mundo exento de reflexividad ni podría haber generado sujeto alguno, ni sería inteligible para ningún sujeto.

Esta epistemología de la reflexividad no nos lleva tanto al abandono del presupuesto de objetividad como, tal como indica Navarro (1989: 93), a su profundización o generalización, ya que la objetividad reflexiva «desborda el objeto e incluye en su radio de acción al sujeto, que así debe dar cuenta de sí 
mismo en los términos de los que es producto: la propia objetividad por él constituida». Ello conlleva la objetivación de la subjetividad, apuntando al desvelamiento de las categorías no pensadas (autorreflexividad), al constituir las precondiciones de las prácticas científicas, por lo que la práctica científica implica objetivación mutua entre el sujeto investigador (objetivador) y la realidad investigada (objetivada-objetivadora).

El retorno reflexivo sobre la práctica científica y sus instrumentos responde al fin de explicitar los presupuestos implícitos de la posición del observador, esto es, a determinar las condiciones de posibilidad del conocimiento del objeto por el sujeto, al ser el objeto no algo exterior e independiente del sujeto, sino producto de la actividad objetivadora del sujeto. En consecuencia, no es difícil de ver lo que implica este enfoque: las objetividades contempladas por la ciencia no están libres de limitaciones epistémicas intrínsecas. La ciencia trata con objetividades cercadas por limitaciones epistémicas intrínsecas, por lo que aquéllas objetividades no son susceptibles de ser concebidas como realidades absolutas, autosuficientes y completamente independientes de la acción del sujeto epistémico. Éste es el logro de la cibernética de segundo orden.

\section{La cibernética de segundo orden}

La perspectiva cibernética constituye una alternativa a la epistemología clásica (que presupone la existencia de una realidad «externa»), al sostener que «construimos» la realidad antes de formar el teatro de nuestras experiencias. Originariamente, la tarea principal de la primera revolución cibernética (alineada con la propuesta sistémica) fue la creación de un lenguaje interdisciplinario, basado en la noción de información-organización, que permitió fundar una nueva disciplina de la mente, que pone «el acento en la continuidad de lo viviente (lo biológico, lo cultural y sus extensiones tecnológicas)» (Pakman, 1994: 35). Fue dicho acento en la continuidad lo que hacía de toda teorización cibernética una meditación sobre la ecología, complementaria de su interés por la autonomía, entendidas ambas nociones como anverso y reverso de la noción de organización. Proveer este lenguaje ha sido el aporte de la cibernética, y bajo la premisa de que el ser y el hacer son inseparables en el contexto de una visión biológica de lo que significa ser «humano», capaz de crear continuamente su representación de la realidad a través del proceso de su vida.

La revolución epistemológica actual plantea el paso de la cibernética de primer orden o de los sistemas observados (que sitúa al observador en el exterior del sistema observado) a la cibernética de segundo orden o de los sistemas observadores (que incluye al observador en el sistema observado). En la cibernética de segundo orden la tradicional distinción entre objetividad y subjetividad se cuestiona hasta el punto de afirmar que la ciencia, más que fundarse en el presupuesto de objetividad (el objeto es objetivo, es exterior al sujeto y no ejerce ninguna acción objetivadora), se funda en el presupuesto de reflexividad: un objeto sólo es definible en relación con un sujeto. Esto es, cualquier sistema está necesariamente formado por dos elemento: un sujeto y la realidad que ese 
sujeto intenta objetivar. Este planteamiento, que cuestiona abiertamente el principio de objetividad, "transformó a la cibernética en una epistemología, una disciplina que tenía algo que decir no solamente sobre la estructura ontológica de la realidad sino sobre el conocimiento de esa realidad, sus límites y posibilidades, sus dificultades y condicionamientos». Así, la cibernética «se volvía sobre sí misma y usaba sus conceptos para ver a los usuarios de dichos conceptos y la relación que a través de esos conceptos establecían con su entorno» ${ }^{1}$ (Pakman, 1994: 26).

La epistemología de segundo orden traslada la atención de los «sistemas observados» a los «sistemas observadores», al considerar que toda noción cibernética (y toda noción es cibernética) es dependiente del observador. Así, cuando se adopta un punto de vista reflexivo, la actividad del sistema objeto y la actividad objetivadora del sujeto deben pensarse como procesos coexistentes. Ahora bien, Navarro (1999: 88) considera que «si entre estos dos tipos de procesos coexistentes se generan necesariamente interferencias es porque los mismos no son ni mutuamente reducibles ni completamente separables. Por una parte, ni la actividad del sistema objeto es independiente (y, por lo tanto, separable) de la actividad objetivadora del sujeto, ni ésta es independiente de aquélla. Por otra parte, ni la actividad objetivadora del sujeto es reducible a la objetividad característica del sistema objeto, ni esta objetividad es reducible a tal actividad».

Frente a la ciencia clásica que trata de conocer los objetos expulsando de la realidad sus dos entidades más características (el sujeto y los valores), la ciencia no clásica centra su atención en las acciones de los sujetos, esto es, reincorpora a la realidad el sujeto y los valores; de manera que la primera cibernética lo efectuó de forma restringida y la segunda cibernética, de modo generalizado.

\section{La epistemología de G. Bateson}

Bateson (1994) se plantea la cuestión de que si aceptamos que nuestro conocimiento del universo determina la relación que tenemos con él, inevitablemente debemos preguntarnos cómo adquirimos nuestro conocimiento. Si tal cuestión resulta de interés, argumenta Bateson, haremos bien en estudiar biología y especialmente esa rama de la biología que denominamos «epistemología». Preservar la naturaleza biológica del mundo y de nuestra relación con él constituye el argumento principal de la epistemología batesoniana ${ }^{2}$. Para este

1. Son conceptos de segundo orden aquéllos que tienen capacidad autorreferencial: «el fin (teórico) de la cibernética no clásica es el estudio de las relaciones entre la acción y sus efectos objetivantes, o lo que es equivalente, el estudio de las relaciones entre estos efectos a través de la acción y entre las acciones a través de estos efectos. Eso implica que la "idea de objetividad" de la cibernética no clásica no puede ser objetivista (en el sentido de objetivada de una vez para todas [...] Desde un punto de vista genuinamente no clásico los procesos objetivantes no son contemplados desde una perspectiva reduccionista, aunque relativista. Son concebidos desde un punto de vista productivo y reflexivo» (Navarro, 1990: 44-45).

2. Lo que le lleva a atribuir un cierto carácter sagrado a la organización biológica del mundo. 
autor, epistemología es aquella ciencia cuyo objeto son las reglas del pensamiento: «no hay cosas que estudiar, sólo se estudian ideas, sólo las ideas de las cosas». Particular epistemología que Bateson caracteriza como una rama de la historia natural al considerar que constituye «un rasgo universal de la percepción humana, un elemento de los fundamentos de la epistemología humana, el hecho de que quien percibe deba percibir sólo el producto de su acto perceptivo. No debe percibir los medios en virtud de los cuales se creó ese producto. El producto mismo es una especie de obra de arte» (Bateson, 1994: 33 y 245-287).

Para este autor, las teorías sólo son construcciones mentales, modelos, y en ningún caso hay que tomar la explicación de un hecho por el propio hecho. El científico, lejos de poder mantener una posición de observador exterior, está implicado en sus teorías científicas como el artista en sus cuadros. Éste es el mensaje de Bateson, y por extensión del constructivismo de la escuela de Palo Alto, que supone una transformación de los tradicionales puntos de vista del paradigma de la ciencia clásica ${ }^{3}$, que postulaban la separación entre el individuo y el entorno y sus corolarios, esto es, la pretensión de que el investigador estudia objetivamente la realidad desde el exterior. Esto implica que la objetividad deriva de la acción de los sujetos por sus efectos objetivantes.

La epistemología batesoniana parte de dos premisas básicas: en primer lugar, la idea de que toda vida mental tiene relación con el cuerpo físico ${ }^{4} \mathrm{y}$, en segundo lugar, la idea de «que ver el mundo ateniendo a las cosas es una deformación sustentada por el lenguaje y que la visión correcta del mundo es aquélla que atiende a las relaciones dinámicas que rigen el crecimiento» (Bateson, 1993: 390). De ahí que pensar en las relaciones, en las relaciones entre relaciones, se estipula como básico en un esquema epistemológico que afirma que el eje central de la existencia humana es su ocurrencia en un dominio lingüístico cognitivo, al ser todos los «fenómenos», literalmente, apariencias ${ }^{5}$, por lo que nunca nos enfrentamos con la realidad en sí, sino sólo con imágenes o concepciones de la realidad, es decir, con interpretaciones, por tanto, es absurdo discutir sobre qué es «realmente» real.

3. Prigogine y Stengers (1983) señalan que la ciencia clásica está constituida como producto de una cultura, en contra de ciertas ideas dominantes en dicha cultura (en particular el aristotelismo, pero también la magia y la alquimia). Incluso podría decirse que está constituida en contra de la naturaleza, puesto que niega la complejidad y el devenir, alegando un mundo eterno y conocible regido por un pequeño número de leyes sencillas e inmutables. La ciencia clásica tiende a describir la verdad única del mundo a partir del principio de que todo lo que existe puede ser deducido, lo que equivale a asumir la esencia de una objetividad maestra.

4. «No podemos salir del mundo determinado por nuestro cuerpo y nuestro sistema nervioso. No existe otro mundo excepto el que experimentamos por medio de estos procesos, procesos que son premisas para nosotros y hacen del nosotros lo que somos. Nos encontramos dentro de un dominio cognoscitivo del cual no podemos salir, o decidir dónde comienza o cómo se crea» (Varela, 1998: 104-105).

5. Para Navarro (1999) la sociedad humana está elaborada a través de procesos reflexivos entre consciencias. 
El lenguaje produce el efecto de referirnos a las propiedades de las cosas como si fueran externas al proceso de representación, cuando de hecho sólo existen como distinciones específicas para el observador. Es importante recordar, advierte Bateson (1994: 185-186), que los mapas lingüísticos con los que trabajamos distan mucho de corresponder al «territorio»: «no sólo estamos obligados, como todas las criaturas, a tratar con ideas de cocoteros antes que con los cocoteros mismos, con los cocoteros reales, sino que además podemos encontrarnos en una isla trópica e imaginar robles, decir mentiras sobre los robles, jugar con ellos o, por simple transformación lingüística, formular una proposición sobre el roble o sobre el cocotero y convertirla en su opuesta. El lenguaje humano es desgraciadamente como el dinero [...] es tan flexible que puede falsificarse».

Para Bateson (1994: 32-33) el mapa no es el territorio. El puente entre mapa y territorio es la diferencia ${ }^{6}$. Así, el autor parte de la comparación entre diferencias, y concretamente de la distinción entre Pleorema (el mundo de la física) y Creatura (el mundo del espíritu), que ilustra la diferencia que hay entre el mapa y el territorio: "cuando distinguimos Creatura y Pleorema en virtud de un primer acto primario de distinción, fundamos la ciencia de la Epistemología, las reglas del pensamiento. Y nuestra Epistemología es una buena epistemología en la medida en que las regularidades del Pleorema pueden traducirse correcta y apropiadamente a nuestro pensamiento y en la medida en que nuestra lógica, la ecología, el pensamiento, el amor, el odio y toda la organización humana (todo aquello que exige diferentes tipos de descripción de aquéllos que empleamos para describir el mundo material inanimado) puede desarrollarse y situarse en ese primer paso de la Epistemología como la ciencia que estudia el proceso de conocer: la interacción de la capacidad de responder a las diferencias, por un lado, con el mundo material en que de algún modo se originan esas diferencias, por otro lado. Debemos considerar, pues, una superficie o línea de encuentro entre el Pleorema y la Creatura».

\section{El conocimiento del conocimiento}

El desarrollo de la mecánica cuántica y de la teoría de la relatividad asestó un golpe terrible a uno de los pilares de la ciencia clásica: la objetividad del observador $^{7}$. En este sentido, Ibáñez (1994) advierte que en la teoría cuántica nada existe si no es observado. Sólo puede existir un universo que sea capaz de producir observadores, y las condiciones de la observación tienen su fundamento

6. La diferencia es algo abstracto y, por tanto, universal. Incluso «antes de poder hablar de autoorganización hay que suponer ya cierta diferencia entre una unidad (o un sistema) y su medio (o su entorno, si lo prefieren) en parte como en la relación figura/fondo» (Varela, 1983: 47).

7. Para Luhmann (1996: 51), «la ciencia ya no se puede concebir más como representación del mundo. Produce una exploración de posibles construcciones que se pueden introducir en el mundo y producen el efecto de la forma, es decir, producen una diferencia». 
en la existencia de información, al ser la realidad una creación del sistema nervioso: «las propiedades de la realidad son propiedades de nuestra representación de la realidad y no propiedades de los objetos» (Ibáñez, 1985: 154). El punto de partida son, pues, las relaciones. Así, la sociedad, para Luhmann, es una relación de relaciones. Según Dupuy $(1995 ; 1998)$, la clave de lo social no es la economía sino la religión, esto es, las relaciones entre sujetos. La religión es lo que religa. Lo que religa es bien un jefe o bien el pánico. Lo que sucede es que la solución del jefe es estable y la del pánico, no. Pero para este autor, el pánico revolucionario siempre acaba tomando la forma de un jefe: Napoleón o Stalin, pero, para este autor, el pánico abre la posibilidad de unas relaciones sociales basadas en la autonomía de los sujetos.

Cibernéticamente, lo que caracteriza a los sistemas dotados de clausura organizacional es su organización autopoiética, ya que no es el entorno el que actúa sobre la organización sino que es la organización la que se autoproduce y genera sus propias organizaciones. Un modelo de sistema autónomo es el ser vivo o hablante, que es organizacionalmente cerrado (autoorganizado) e informacionalmente abierto (aprende/conoce). Pero al intentar conocer el conocer nos encontramos con nuestro propio ser; un ser que es constitutivamente social, pues lo humano no puede existir fuera de lo social. Para Maturana y Varela (1991: 158) los sistemas sociales humanos existen como unidades para sus componentes en el dominio del lenguaje. Así, la identidad de los sistemas sociales humanos depende a la vez de la adaptación de los seres humanos como organismos y de su adaptación como componentes de los dominios lingüísticos que constituyen. En consecuencia, «mientras que para el operar de un organismo lo central es el organismo y de ello resulta la restricción de las propiedades de sus componentes al constituirlo, para el operar de un sistema social humano lo central es el dominio lingüístico que generan sus componentes y la ampliación de las propiedades de éstos, condiciones necesarias para la realización del lenguaje, que es su dominio de existencia. El organismo restringe la creatividad individual de las unidades que lo integran, pues éstas existen para éste; el sistema social humano amplía la creatividad individual de sus componentes, pues éste existe para éstos».

Lo central del fenómeno social humano es que se da en el lenguaje, y lo central del lenguaje es que sólo en él se dan la reflexión y la autoconciencia. Afirma Maturana (1996: 16-17) que en un sentido antropológico el lenguaje es «el origen de lo humano propiamente tal a la vez que su caída y liberación. El lenguaje saca la biología humana del ámbito de la pura estructura material e incluye en ella el ámbito de la estructura conceptual, al hacer posible un mundo de descripciones en el que el ser humano debe conservar su organización y adaptación. Así, el lenguaje da al ser humano su dimensión espiritual en la reflexión, tanto de la autoconciencia como de la conciencia del otro. Pero el lenguaje es también la caída del ser humano, al permitir las cegueras frente al ser biológico que traen consigo las ideologías descriptivas de lo que debe ser».

Maturana (1996) postula dos caminos para el observador científico: 1) el de la objetividad a secas y 2) el de la objetividad entre paréntesis. Estos dos 
caminos no son la antinomia objetivo/subjetivo: la objetividad entre paréntesis no dice subjetividad, sino que dice que yo no asumo poder hacer referencia a entidades, a objetos, independientes de mí. La objetividad entre paréntesis sitúa el conocimiento en la vida humana. Se trata de elaborar una "ontología del observar» fundada en la reformulación de las condiciones de constitución del observar, esto es, en explicar qué es lo que constituye el observar como fenómeno biológico, qué es lo que constituye el conocer como fenómeno biológico.

El conocimiento del conocimiento obliga, pues lo que la biología nos muestra es que la unicidad de lo humano, su patrimonio exclusivo, está en darse en un acoplamiento estructural social donde el lenguaje tiene un doble rol: «por un lado, el de generar las regularidades propias del acoplamiento estructural social humano $^{8}$, que incluye, entre otros, el fenómeno de las identidades personales de cada uno y, por otro lado, el de constituir la dinámica recursiva del acoplamiento estructural social que produce la reflexividad que da lugar al acto de mirar con una perspectiva más abarcadora, al acto de salirse de lo que hasta ese momento era invisible o inamovible, permitiendo ver que como humanos sólo tenemos el mundo que creamos con otros. Y es que no es el conocimiento, sino el conocimiento del conocimiento lo que obliga. No es el saber que la bomba mata, sino lo que queremos hacer con la bomba lo que determina el que la hagamos explotar o no. Esto, corrientemente, se ignora o se quiere desconocer para evitar la responsabilidad que nos cabe en todos nuestros actos cotidianos, ya que todos nuestros actos, sin excepción, contribuyen a formar el mundo en que existimos y que validamos, precisamente, a través de ellos, en un proceso que configura nuestro devenir. Ciegos ante esta trascendencia de nuestros actos, pretendemos que el mundo tiene un devenir independiente de nosotros que justifica nuestra irresponsabilidad en ellos, y confundimos la imagen que buscamos proyectar, el papel que representamos, con el ser que verdaderamente construimos en nuestro diario vivir» (Maturana y Varela, 1991: 208-210).

Las teorías científicas son el producto de seres inscritos en el mundo por ellos explorado. La ciencia, en tanto que dominio cognitivo, es un dominio de explicaciones esenciales. Y lo es, no porque las explicaciones científicas sean objetivas o verdaderas en un sentido trascendental, sino porque surgen y permanecen en nuestro dominio de constitución como sistemas vivientes. De

8. «El concepto de clausura operacional es la consecuencia de la tesis mediante la cual ningún sistema puede operar fuera de sus propios límites. Cada sistema tiene naturalmente un entorno y permanece dependiente de la compatibilidad con él mismo, lo que en la noción de Maturana se incida como acoplamiento estructural: la «relación» entre un sistema y los presupuestos del entorno, que deben presentarse para que pueda continuar dentro de su propia producción o autopoiesis. Todo sistema siempre se halla o se encuentra adaptado a su entorno, en otro caso no existiría. Un sistema, si existe y opera, está adaptado siempre al entorno. Al subrayar la clausura del sistema ciertamente no se quiere negar la relevancia del entorno: la ya clásica contraposición entre sistemas abiertos y sistemas cerrados está superada con la afirmación de que es la clausura la condición para la apertura del sistema» (De Toca, 1997: 86). 
hecho, como afirman Prigogine y Stengers (1983: 40 y 37-38), no existe en la actualidad un modo canónico de abordar el problema de la ciencia: «solamente conocemos el precio inaceptable que han tenido que pagar quienes han intentado "purificar" el tema, olvidando que la descripción de la actividad científica no puede separarse sin conflicto de la descripción del mundo al que pertenece».

Pero, ¿de qué hipótesis de la ciencia clásica se ha liberado la ciencia actual? Fundamentalmente, de aquéllas que se basan en la convicción «de que el mundo microscópico es simple y está gobernado por leyes matemáticas simples, lo cual significa que la labor de la ciencia consiste en superar las apariencias complejas y reducir los diversos procesos naturales a un conjunto de efectos de dichas leyes. Este concepto de los objetos científicos conlleva a una discriminación entre lo que en la naturaleza se supone corresponde a una realidad «objetiva» y lo que se considera ilusorio, ligado a nuestra propia subjetividad. De hecho, las leyes matemáticas simples a las cuales, según se cree, están sujetos los comportamiento elementales y que construiría la verdad última del universo, casi siempre han sido concebidas sobre el modelo general de las leyes dinámicas». Estas leyes describen el mundo en términos de trayectorias deterministas y reversibles. Desde la aparición de la mecánica cuántica, sin embargo, la idea de la simplicidad del microscopio se ha vuelto insostenible al estar «en un mundo indiscutiblemente aleatorio, en un mundo en el que la reversibilidad y el determinismo son casos particulares y en el que la irreversibilidad y la indeterminación microscópicas son la regla» (Prigogine y Stengers, 1983: 37-38). Por otra parte, como señalan ambos autores, en los acontecimientos naturales el tiempo corre en una sola dirección. Podemos, por un lado, recordar el pasado, pero, por otro, no podemos recordar el futuro. He aquí la flecha del tiempo, la mensurabilidad del tiempo. Estamos en el tiempo, estamos, por tanto, dentro de los mismos hechos que investigamos.

En su constitución como dominio cognitivo, la ciencia es el domino de las explicaciones y declaraciones que los científicos generan mediante la aplicación del criterio de validación del conocimiento, que se halla en conexión con la reformulación explicativa de la praxis experiencial y que debe satisfacer, para Maturana (1996: 74-75), las siguientes cuatro operaciones: «i) La presentación de la experiencia (fenómeno) que ha de explicarse en términos de lo que un observador tipo tiene que hacer en su dominio de experiencias (práctica de vida) para experimentarla. ii) La reformulación de la experiencia (fenómeno) que ha de explicarse en la forma de un mecanismo generativo que, si realizado por un observador tipo en su dominio de experiencias, le permitiría, como consecuencia de su operación, tener en su dominio de experiencias la experiencia que ha de ser explicada como está presentado en el punto i. iii) La deducción, a partir de la operación del mecanismo generativo propuesto en ii), al igual que de todas las coherencias operacionales del dominio de experiencias de un observador tipo implicadas por ésta, de otras experiencias que un observador tipo debe tener a través de la aplicación de esas coherencias operacionales y de las operaciones que él o ella debe realizar en su dominio de 
experiencias para tenerlas. iv) La experiencia, de un observador tipo, de las experiencias (o fenómenos) deducidas en iii) por medio de la realización del observador tipo en su dominio de la experiencia de las operaciones también deducidas en iii)».

La ciencia, en tanto que dominio de las explicaciones que los científicos generan, configura al científico al operar como científico y, como tal, el científico aborda la ciencia con la explicación y comprensión de su experiencia humana, «no con la explicación y comprensión de la naturaleza o la realidad como si éstas fueran dominios objetivos de existencia independientes de lo que hacemos. La experiencia humana está libre de contenido. En nuestra experiencia no nos encontramos con cosas, objetos o naturaleza como entidades independientes, como podría parecernos en la ingenuidad de la vida diaria; vivimos en la experiencia, en la práctica de vivir de seres humanos en el flujo de ser sistemas vivientes en el lenguaje como algo que nos sucede en nuestro interior y a nosotros mismos mientras lo lenguajeamos. Ésta es la razón por la que, mientras que los científicos explicamos nuestra experiencia como seres humanos al reformularla con elementos suyos a través de la aplicación del criterio de validación de explicaciones científicas, nos encontramos generando ciencia como un dominio cognitivo que no nos extrae de la experiencia y que nos mantiene en el lenguaje. Los seres humanos existimos en el lenguaje, y nuestra experiencia como seres humanos se lleva a cabo en el lenguaje en un flujo de coordinaciones consensuales de acciones que ponemos de manifiesto en el lenguaje» (Maturana, 1996: 95-96).

Conocer cómo conocemos, esto es, cómo se validan nuestras apreciaciones cognitivas, pertenece al ámbito de la vida cotidiana, ya que las explicaciones científicas surgen como acciones humanas reales dentro del dominio «de experiencias de observadores tipo individuales, y son válidas como tales en una comunidad formada por éstos, aunque cada observador tipo vive sus experiencias en la completa soledad de su determinismo estructural como sistema viviente. Esta situación no constituye una contradicción debido a que el formar parte de la comunidad de observadores tipo no depende de la capacidad individual de referirse a una realidad objetiva independiente que el observador tipo como sistema viviente no puede hacer, sino de la participación consensual en el dominio de las explicaciones científicas como un dominio de coexistencia» (Maturana, 1996: 76-77). Por lo tanto, añade el autor, "sólo aquellos observadores que pueden participar con otros observadores, y a su entera satisfacción, en la realización del criterio de validación de explicaciones científicas, y, además, aceptan esto como su único criterio de validación para sus explicaciones, son científicos (observadores tipo) y miembros de la comunidad de científicos. Los observadores que por una o otra razón no pueden o quieren hacer esto, son descartados como observadores tipo o científicos por la comunidad de científicos, o no son considerados para nada».

En definitiva, nos hallamos ante una lógica autorreflexiva que parte del sujeto observador y de su papel en el proceso de conocimiento. Todo lo que 
es, es un constructo del observador. Por tanto, la relación de conocimiento no es una relación entre un sujeto y un objeto preexistentes: sujeto y objeto son productos de la relación. Debemos distanciarnos de la idea de entidades aisladas y autosuficientes para centrarnos en las nociones de relación y de organización. Esta perspectiva de estudio es la que adopta el enfoque sistémico, y concretamente la metodología constructivista de la cibernética de segundo orden o cibernética de los sistemas observadores, que estipula como central el principio autorreferencial de inclusión explícita del observador y de sus instrumentos de observación en la descripción de la observación: «una descripción (del universo) implica aquél que describe (que lo observa). Lo que necesitamos ahora es la descripción de «aquél que describe» o, dicho de otro modo, necesitamos tener una teoría del observador». En contra del postulado epistemológico básico que afirma la existencia de un "mundo objetivo» invariante a la descripción para, posteriormente, intentar «escribir su descripción, sentimos ahora el reto de desarrollar un "mundo subjetivo" invariante a la descripción, es decir, un mundo que incluya al observador: éste es el problema» (Von Foerster, 1991: 63-64). Y, sin embargo, la tradición clásica continúa reclamando la validez de una epistemología que ha sido formulada en términos de "¿cómo conocemos?», en lugar de una epistemología acerca de «¿qué conocemos?».

\section{El constructivismo}

El constructivismo, en tanto que epistemología de la nueva cibernética, revela un esquema epistemológico maximizador de la interacción entre el observador y lo observado. De ahí que la autoobservación se muestre capaz de plantear la constitución de una ciencia social con sujetos, no meramente reproductora de un estado de hechos a partir de su descripción externa. El constructivismo identifica al observador y la observación, pues ambos son operaciones dentro del lenguaje. Por esta razón, y dado que la existencia está ligada a nuestras distinciones en el lenguaje, los «sistemas sociales humanos son sistemas de coordinaciones de acciones en el lenguaje; esto es, son redes de conversaciones. Consecuentemente, los diferentes sistemas sociales humanos, o sociedades, difieren en las características de las diferentes redes de conversaciones que los constituye» (Maturana, 1996: 74).

Somos nosotros, los seres humanos, quienes "producimos un "mundo objetivo" a través del uso de nuestros propios cambios de estado como descriptores que especifican los objetos que lo constituyen" (De Toca, 1997: 154). En consecuencia, la realidad es una proposición explicativa y la razón, un fenómeno del operar dentro del lenguaje, por lo que, como afirma Maturana (1996: 35), «la racionalidad no es una propiedad del observador, u observadora, que le permite conocer algo que existe independientemente de lo que él o ella hacen, sino que es la operación del observador, u observadora, de acuerdo con las coherencias operacionales de lenguajear en un dominio determinado de la realidad. Y, consecuentemente, hay tantos 
dominios de racionalidad como dominios de realidad produce el observador, u observadora, en su praxis de vivir en tal calidad». Además, las explicaciones, al tener lugar en la praxis de vivir del observador, constituyen experiencias. Sin embargo, las explicaciones en tanto que experiencias son experiencias de segundo orden, en el sentido que son reflexiones del observador en su praxis de vivir en el lenguaje acerca de su praxis de vivir. En este contexto, "la realidad no es una experiencia, es un argumento dentro de una explicación. En otras palabras, la realidad surge como una proposición explicativa de nuestra experiencia de las coherencias operativas de nuestra vida cotidiana y de nuestra vida técnica, al vivir nuestra vida cotidiana y nuestra vida técnica» (Maturana, 1996: 31).

Las nociones de "complementariedad», «contingencias», «sistemas irreversibles», "estructuras emergentes», "diversidad de comportamientos propios», "autoorganización», etc., despliegan una nueva imagen de la ciencia que no se orienta por la metáfora maquínica, sino, en contra, por la metáfora organísmica. De aquí que dado que el observador, el fenómeno observado y el proceso de observación constituyen una totalidad, los cibernéticos y/o científicos de segundo nivel se observan cada vez que observan un sistema. Por ello se afirma "que las explicaciones científicas son proposiciones de mecanismos que generan los fenómenos que el investigador trata de explicar, y ese mecanismo consiste en un sistema determinado estructuralmente: un conjunto de elementos conectados de manera tal que lo que pasa con ese conjunto de elementos depende de cómo está hecho. Ahora bien, de esta manera es como nos manejamos todos en la vida cotidiana» (De Toca, 1997: 156).

El constructivismo considera que los sistemas son irreversibles, inestables, impredecibles, envueltos en procesos de autoorganización alejada del equilibrio. Es, pues, una epistemología que asume que la realidad está biológica y socialmente (lingüísticamente) construida, pues allí donde los «organismos requieren un acoplamiento estructural no lingüístico entre sus componentes, los sistemas sociales requieren componentes acoplados estructuralmente en dominios lingüísticos donde los componentes puedan operar con lenguaje y ser precisamente observadores. En consecuencia, lo central para el operar de un sistema social es el dominio lingüístico que generan sus componentes y la ampliación de las propiedades de éstos, condición necesaria para la realización del lenguaje, que es el dominio de existencia». Para el constructivismo cualquier descripción del mundo es una invención en la medida que se realiza desde la perspectiva de observación del observador como participante constructor de realidades. Así, el constructivismo y/o cognitivismo pone en tela de juicio «el supuesto más arraigado de nuestra tradición científica: que el mundo tal como lo experimentamos es independiente de quien lo conoce. En cambio, si estamos obligados a concluir que la cognición no se puede entender adecuadamente sin sentido común, el cual no es otra cosa que nuestra historia corporal y social, la inevitable conclusión es que conocedor y conocido, sujeto y objeto, se determinan el uno al otro y surgen simultáneamente. En tér- 
minos filosóficos: el conocimiento es ontológico»9 (Varela, 1998: 96). De aquí que «la problemática epistemológica se plantea hoy en términos de un encuentro y una fecundación mutua entre el saber biológico y el saber psicosociológico: todos los observadores son organismos vivos e individuos en una sociedad, hay que descifrar lo que tiene el observador de inconsciente como ser vivo (lo vivo de la ciencia), y lo que tiene el observador de inconsciente como ser social (lo social de la ciencia). Se impone un cruce de perspectivas entre la biología y la antropo-(psico)-sociología: no se trata de reducir una a otra, ni tampoco de integrarlas a ambas en una perspectiva más compleja, sino de descubrir las singularidades de una y otra haciendo reflexionar a cada una sobre cada otra» (Ibáñez, 1985: 149).

Si se acepta que la presencia del observador (directamente o a través de instrumentos de observación) altera la observación, concluiremos que el mérito de las actuales ciencias cognitivas es haber descubierto que el conocimiento no puede explicarse como una especie de espejo de la naturaleza, sino que más bien existe una implicación mutua entre quien conoce y lo que es conocido. En cada campo científico el objeto y el sujeto se configuran en virtud de su propia interacción constructiva. Así, y de acuerdo con la física cuántica, las antiguas ideas sobre la observación quedan sutilmente minadas. Debemos esforzarnos por rechazar toda pretensión de erigirnos en observadores externos.

Por tanto, si el observador y lo observado forman parte del mismo objeto descrito, la convergencia entre el sujeto cognoscente y el objeto por conocer no puede sustraerse del principio de reflexividad, que propicia una mejor y más profunda solidez del corpus científico generado. La superioridad del principio de reflexividad se fundamenta en la naturaleza autorreferente de los sistemas sociales, esto es, en la capacidad que desarrollan los sistemas para tomar conciencia de sí y delimitarse respecto a un entorno o ambiente. A través de tal delimitación los sistemas acceden a la autoobservación; sin ella los sistemas no son capaces de generar todos los componentes que precisan para sus operaciones, esto es, no son capaces de llevar a cabo sus procesos autopoiéticos. Ello indica, según Luhmann, que toda observación (al depender de los medios de observación) está necesariamente mediada por los sistemas sociales. Lo que cuenta en la cibernética de segundo orden son los contextos en los que tiene lugar la observación y la actuación de los sistemas que observan las observaciones de otros sistemas, que observan y actualizan la sociedad desde sus respectivos ángulos concretos. Desde esta perspectiva, la tarea de la sociología

9. En la misma línea, Maturana (1996: 96) argumenta que el constructivismo es una teoría del comprender: «un aspecto central de hacer ciencia tiene que ver con nuestra búsqueda por la comprensión de nuestra experiencia como seres humanos. Y por comprensión quiero decir la experiencia de adoptar una operacionalidad de reflexión en el lenguaje en donde podamos saber lo que sabemos dentro de las circunstancias de constitución del lenguaje. Para poder reflexionar, tenemos que dejar ir a lo que constituimos en nuestras distinciones como un objeto de nuestra reflexión, para que podamos contemplarlo, pero lo que debemos hacer sin temer perder lo que dejamos ir, para que lo podamos ver sin opacarlo con el vínculo que tenemos con él». 
estriba en responder a la siguiente pregunta: ¿cómo se comunica la observación del mundo en el mundo?

\section{Conclusión}

Reconocer que el investigador pertenece al mismo orden de realidad que investiga, funda la posibilidad de conocimiento al extender el campo de observación a su propia subjetividad. Hay un espacio de comunicación entre el investigador individual y la sociedad que le incluye, no como producto o reflejo, sino como parte de lo mismo. Este espacio de comunicación es el lenguaje, que es a la vez un instrumento y un objeto de la investigación social. Por ello, la objetividad científica se constituye en ruptura con los objetos preconstituidos por el lenguaje común. El lenguaje tiene «un problema particularmente dramático para el sociólogo: y es que es un inmenso depósito de preconstrucciones naturalizadas, por tanto ignoradas como tales construcciones, y que funcionan como instrumentos inconscientes de construcción social». En las ciencias sociales las rupturas epistemológicas son rupturas sociales, esto es, "rupturas con las creencias fundamentales de un grupo, y, a veces, con las creencias fundamentales del cuerpo profesional, con el corpus de creencias compartidas que fundamentan la communis doctorum opinio» ${ }^{10}$ (Bourdieu, 1997: 210).

Frente a los teóricos puros y a los metodólogos puros, esto es, frente a las distorsiones metodológicas y a las desviaciones especulativas, apostar por una epistemología renovada permite preguntarse qué es hacer ciencia en las condiciones y/o situaciones concretas de la práctica científica. $\mathrm{O}$, más correctamente, «tratar de saber qué hace el científico, sepa éste o no lo que hace, no es sólo interrogarse sobre la eficacia y el rigor formal de las teorías y de los métodos, es examinar a las teorías y los métodos en su aplicación para determinar qué hacen con los objetos y qué objetos hacen» (Bourdieu y otros, 1976: 25). Este examen impone la jerarquía epistemológica de los actos científicos, que subordina la comprobación a la construcción del objeto y la construcción a la ruptura con los objetos preconstruidos.

El problema de la objetividad es una cuestión epistemológica. En primer lugar, porque la observación es un proceso activo de producción de datos que supone una intervención sobre lo observado, y que por tanto lo modifica; $y$, en segundo lugar, porque la escala y/o medida con la que se pretende dimensionar algo externo al sujeto jamás es aséptica y/o neutra, sino que siempre representa un valor. En este sentido, Crespán (1986: 118) destaca que «en la historia de la humanidad, la fijación de medidas ha sido y continúa siendo un atributo del poder. Medir o ser medido representan posiciones diversas en las acciones de poder y todo es susceptible de medición excepto el propio poder,

10. «El discurso epistemológico (que se refiere a la operación de conquista o captura) constituye un metalenguaje de los discursos metodológicos (que se refiere a la construcción) y tecnológico (que se refiere a la constatación); el discurso metodológico es un metalenguaje del discurso tecnológico» (Ibáńez, 1985: 209). 
que siempre resulta inconmensurable. De alguna manera podemos decir que el sometimiento a medida de los objetos, y no digamos ya la construcción de tal medida, resulta un ejercicio de poder que puede resultar tanto más despótico cuanto menos respete las peculiaridades y el contexto del objeto».

\section{Bibliografía}

Bateson, G. (1985). Pasos hacia una ecología de la mente. Buenos Aires: Carlos Lohlé. - (1993). Unidad Sagrada. Barcelona: Gedisa.

BATESON, G.; BATESON, M.C. (1994). El temor de los ángeles. Barcelona: Gedisa.

BOURDieu, P. (1997). Razones prácticas. Sobre la teoría de la acción. Barcelona: Anagrama. Bourdieu, P. y otros (1976). El oficio de sociólogo. Madrid: Siglo XXI.

CRESPÁN, J.L. (1986). «De la metodología y de las especies dimisionarias». Papers, 26.

De Toca, S. (1997). Psicologías cognitivas. Ciencias y tecnologías. Madrid: Biblioteca Nueva.

DUPUY, J.P. (1995). «En torno a la autodesconstrucción de las convenciones». En WATZLAWICK, P.; KRIEG, P. (comps.). El ojo del observador. Contribuciones al constructivismo. Barcelona: Gedisa.

- (1998). El sacrificio y la envidia. El liberalismo frente a la justicia social. Barcelona: Gedisa.

FOERSTER, H. Von (1991). Las semillas de la cibernética. Madrid: Gedisa.

IbÁÑEZ, J. (1985). Del algoritmo al sujeto. Nuevas perspectivas de la investigación social. Madrid: Siglo XXI.

- (1994). Por una sociología de la vida cotidiana. Madrid: Siglo XXI.

Lummann, N. (1998). Complejidad y modernidad. De la unidad a la diferencia. Madrid: Trotta.

Maturana, H. (1996). I. Fundamentos biológicos de la realidad. La realidad ¿objetiva o construida? Barcelona: Anthropos.

- (1996). II. Fundamentos biológicos del conocimiento. La realidad ¿objetiva o construida? Barcelona: Anthropos.

Maturana, H.; VARELA, F. (1991). El árbol del conocimiento. Las bases sociales del conocimiento humano. Madrid: Debate.

NAVARRO, P. (1989). «Sistemas reflexivos». En REYES, R. (dir). Terminología científicosocial. Aproximación crítica (Apéndice). Madrid: Anthropos.

- (1990). "Ciencia y cibernética. Aspectos teóricos». En IBÁÑEZ, J. (coord.). Nuevos avances en la investigación social, I y II. Barcelona: Proyecto A Ediciones.

- (1990a). «Tipos de sistemas reflexivos». En IBÁN̄EZ, J. (coord.). Nuevos avances en la investigación social, I y II. Barcelona: Proyecto A Ediciones.

- (1999). «Las dos formas de la reflexividad social humana». En RAMOS TORRE, R.; GARCÍA SELGAS, F. Globalización, riesgo y reflexividad. Madrid: CIS.

PAKMAN, M. (1994). «Investigación en grupos familiares. Una perspectiva constructivista». En DelGadO, J.M.; GuTIÉRREZ, J. (coord.). Métodos y técnicas cualitativas de investigación en ciencias sociales. Madrid: Síntesis.

Prigogine, I.; Stengers, I. (1983). La Nueva alianza. Metamorfosis de la ciencia. Madrid: Alianza.

VARELA, F. (1998). «Historia de la reflexividad». En IBÁÑEZ, J. (coord.). Nuevos avances en la investigación social, I y II. Barcelona: Proyecto A Ediciones. 